\title{
EDITORIAL
}

\section{Síndrome agudo respiratorio severo y la respuesta organizada en México: ¿estamos preparados?}

$\mathrm{T}$ ras la emisión de la alerta sobre el síndrome agudo respiratorio severo (SARS) dada por la Organización Mundial de la Salud (OMS), el 15 de marzo de 2003, la Secretaría de Salud, a través de la Dirección General de Epidemiología, informó a las autoridades sanitarias del país acerca de dicha alerta, iniciando así las acciones de respuesta ante este problema emergente de salud pública, con base en la comunicación con organismos internacionales como la OMS y la Organización Panamericana de la Salud y con autoridades sanitarias de Canadá (Health Canada), y los Centros de Prevención y Control de Enfermedades, de Estados Unidos de América.Con la finalidad de organizar las actividades sectoriales se desarrollaron y distribuyeron los lineamientos para la vigilancia, prevención y control del SARS y se estableció, y se ha mantenido, una comunicación directa y constante con Aeropuertos y Servicios Auxiliares, la Dirección General de Aeronáutica Civil, las aerolíneas, los sindicatos de personal de aviación, el Instituto Nacional de Migración, la Administración General de Aduanas, la comunidad médica y la sociedad civil.

La difusión de información a la población debe considerarse como una estrategia central para el éxito de las medidas de prevención y control del SARS. Los mecanismos implantados por la Secretaría de Salud incluyen: información actualizada sobre el padecimiento en el portal de la Dirección General de Epidemiología, presencia constante del vocero único en los medios de comunicación (impresos y electrónicos), difusión de comunicados de prensa oficiales y una línea telefónica gratuita (01800 0044800$)$ a través de la cual se brinda información a la población en general y al personal de salud sobre el padecimiento, las 24 horas del día, los 365 días del año.

Hace más de 22 años que la comunidad internacional se enteró de la aparición de una neumonía atípica en hombres homosexuales, en San Francisco, California. Quién pudo imaginar que esa infeccion respiratoria aguda y severa, ocasionada tambien por un germen poco común, el Pneumocysitis carinii, resultó ser sólo el marcador clínico de la infección por el VIH/ SIDA, y nuestro primer aviso de la epidemia más grande y severa desde la influenza española de principios del siglo XX.

En el caso del SARS, aún no sabemos cuál será su evolución. En el peor escenario, puede ser la primera gran pandemia del siglo XXI, o bien, ante la ausencia de una vacuna o tratamiento específico, puede ser la primera epidemia contenida con medidas de aislamiento respiratorio, o cuarentena, y lavado de manos, eso esperamos.

A la fecha se han detectado 220 casos del SARS en cuatro países de la Región: Canadá con 151, Estados Unidos de América con 66, Brasil con dos y Colombia con un caso. Han ocurrido 26 defunciones, todas en Canadá. En todos estos casos la infección se ha importado de alguno de los países asiáticos afectados; pero en Toronto, Canadá, desafortunadamente ya ha habido transmisión secundaria y, gracias a que las autoridades se montaron en su plan de emergencia para la anticipada pandemia de influenza ésta, aunque no está bajo control, pudo haberse extendido más. En este momento no hay forma de predecir la evolución del padecimiento en las Américas, pero el riesgo de que llegue a México es latente y, de continuar como va la epidemia, es casi inevitable.

Hasta el momento se han presentado en México 20 casos en los cuales se ha sospechado el SARS en los estados de Jalisco, México, Morelos, Nuevo León, Querétaro y el Distrito Federal, sin embargo, ninguno ha llegado a ser sospechoso de acuerdo con la clasificación de la OMS. Todos los casos han sido descartados tras su detección y referencia a los hospitales de con- 
centración designados en cada entidad para ello, de acuerdo con lo establecido en los lineamientos anteriormente señalados.

El 17 de mayo, concluyó en Ginebra la primera reunión de consulta epidemiológica de la OMS, sobre el SARS. A esta reunión asistieron 40 epidemiólogos de 16 países, incluyendo representantes de los países más afectados. Las conclusiones enfatizan las medidas de control de la Secretaría de Salud que se señalan en el texto del artículo Síndrome agudo respiratorio se- vero (SARS): un panorama mundial de la epidemia, de este número de la Revista, y que incluyen identificación, aislamiento y atención de casos sospechosos o probables, vigilancia epidemiológica e información a la población, lo que facilitará las acciones de contención de un brote y evitará la diseminación del padecimiento por nuestro territorio.

Pablo Kuri Morales, ${ }^{*}$ José Ignacio Santos Preciado

\footnotetext{
* Dirección General de Epidemiología, Secretaría de Salud.

‡ Centro Nacional para la Salud de la Infancia y Adolescencia, Subsecretaría de Prevención y Protección a la Salud, Secretaría de Salud.
} 